\title{
Cross Talk with the GAR-3 Receptor Contributes to Feeding Defects in Caenorhabditis elegans eat-2 Mutants
}

\author{
Alena A. Kozlova, ${ }^{1}$ Michelle Lotfi, and Peter G. Okkema ${ }^{2}$ \\ Department of Biological Sciences, University of Illinois at Chicago, Illinois 60607 \\ ORCID ID: 0000-0001-8647-1169 (P.G.O.)
}

\begin{abstract}
Precise signaling at the neuromuscular junction (NMJ) is essential for proper muscle contraction. In the Caenorhabditis elegans pharynx, acetylcholine (ACh) released from the MC and M4 motor neurons stimulates two different types of contractions in adjacent muscle cells, termed pumping and isthmus peristalsis. MC stimulates rapid pumping through the nicotinic ACh receptor EAT-2, which is tightly localized at the MC NMJ, and eat-2 mutants exhibit a slow pump rate. Surprisingly, we found that eat-2 mutants also hyperstimulated peristaltic contractions, and that they were characterized by increased and prolonged $\mathrm{Ca}^{2+}$ transients in the isthmus muscles. This hyperstimulation depends on cross talk with the GAR-3 muscarinic ACh receptor as gar-3 mutation specifically suppressed the prolonged contraction and increased $\mathrm{Ca}^{2+}$ observed in eat-2 mutant peristalses. Similar GAR-3-dependent hyperstimulation was also observed in mutants lacking the ace-3 acetylcholinesterase, and we suggest that NMJ defects in eat-2 and ace-3 mutants result in ACh stimulation of extrasynaptic GAR-3 receptors in isthmus muscles. gar-3 mutation also suppressed slow larval growth and prolonged life span phenotypes that result from dietary restriction in eat-2 mutants, indicating that cross talk with the GAR-3 receptor has a long-term impact on feeding behavior and eat-2 mutant phenotypes.
\end{abstract}

KEYWORDS C. elegans; pharynx; GCaMP3; nicotinic acetylcholine receptor; muscarinic acetylcholine receptor; peristalsis; life span; feeding

OMMUNICATION between motor neurons and their target muscles is crucial for proper muscle contraction and function. This communication is mediated by the neurotransmitter acetylcholine (ACh), which is released from the motor neuron and binds receptors embedded in the muscle cell membrane. In vertebrate skeletal muscle, these receptors are nicotinic ACh receptors (nAChRs), which are homo- or hetero-pentameric, ligand-gated ion channels [reviewed in

Copyright $\odot 2019$ Kozlova, et al.

doi: https://doi.org/10.1534/genetics.119.302053

Manuscript received April 18, 2018; accepted for publication March 14, 2019; published Early Online March 21, 2019.

Available freely online through the author-supported open access option.

This is an open-access article distributed under the terms of the Creative Commons Attribution 4.0 International License (http://creativecommons.org/licenses/by/4.0/), which permits unrestricted use, distribution, and reproduction in any medium, provided the original work is properly cited.

Supplemental material available at Figshare: https://doi.org/10.25386/genetics. 6160355.

1Present address: Department of Psychiatry and Behavioral Neuroscience, NorthShore University Health System, Evanston, IL 60201.

${ }^{2}$ Corresponding author: Molecular, Cell, and Developmental Biology Group (MC567),

Room 4060, Department of Biological Sciences, University of Illinois at Chicago, 900 S.

Ashland Ave., Chicago, IL 60607. E-mail: okkema@uic.edu
Albuquerque et al. (2009)]. In smooth and cardiac muscle, the receptors are typically muscarinic ACh receptors (mAChRs), which are seven-pass transmembrane, $\mathrm{G}$ protein-coupled receptors [reviewed in Wess (2004)]. nAChRs are fast-acting receptors that rapidly and directly depolarize muscle cells to stimulate contraction in response to ACh, while mAChRs act more slowly by activating downstream signal transduction cascades, and can either stimulate or inhibit contraction. A number of inherited and acquired diseases affecting communication at the neuromuscular junction (NMJ) in human skeletal muscle exhibit defects in nAChRs and other proteins required for their function, including myasthenia gravis and congenital myasthenic syndromes [reviewed in Engel et al. (2015), Gilhus (2016)].

We are examining the mechanisms controlling contractions of the pharyngeal muscles of Caenorhabditis elegans. The pharynx is a tubular organ with large muscle cells positioned around a central lumen (Figure 1A) (Albertson and Thomson 1976). The myofilaments in these muscles are oriented radially, so that contraction opens the pharyngeal lumen and relaxation closes it. During feeding, these muscles perform two 

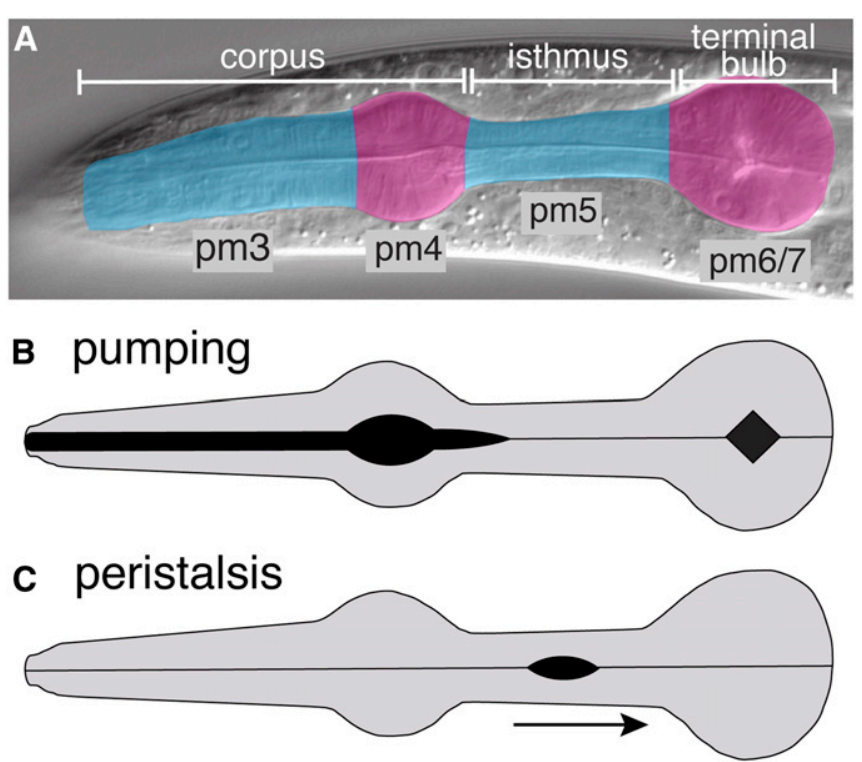

Figure 1 Pharyngeal anatomy and contractions. (A) DIC micrograph of an adult pharynx indicating anatomical regions (white bars) and colored to indicate the location of pharyngeal muscle cells (pm). The pharynx exhibits threefold rotational symmetry, and there are three of the pm3pm7 muscle cells surrounding the central lumen (Albertson and Thomson 1976). pm5 cells extend the length of the isthmus and are the primary focus of this work. (B and C) Diagrams indicating pharyngeal muscle contractions during pumping and peristalsis. The black regions indicate open lumen, and the arrow indicates the direction of the peristaltic contraction.

distinct types of contraction, termed pumping and peristalsis [reviewed in Avery and You (2012)]. Pumping occurs frequently $(\sim 200 / \mathrm{min})$, and it is a simultaneous contraction of muscles in the corpus and anterior isthmus that ingests bacteria into the lumen, and concentrates this material in the anterior region of the isthmus, while at the same time contraction of muscles in the terminal bulb crushes bacteria and expels the debris into the intestine (Figure 1B). Peristalsis occurs relatively infrequently and always following a pump. Peristalsis is a wave-like contraction followed by rapid relaxation traveling through the pm5 muscle that transports a bolus of ingested bacteria from the anterior isthmus to the terminal bulb (Figure 1C). The contractions in the pm5 muscle are remarkably complex. Three pm 5 muscles are arranged around the circumference of the pharyngeal lumen and each of these cells extends through the entire length of the isthmus (Figure 1A) (Albertson and Thomson 1976). During a pump, the anterior portions of the pm5 cells contract to open the lumen in the anterior isthmus, but the posterior halves of these cells remain relaxed. In contrast, during peristalsis, the anterior portion of pm5 remains relaxed, while a wavelike contraction followed by relaxation travels from the center to the posterior isthmus.

Rapid pumping is stimulated by the two MC neurons, which form synapses on the muscles near the junction of the pm 4 and pm5 muscles (McKay et al. 2004), and laser ablation of the MCs results in a slow pump rate (Avery and Horvitz 1989).
The MCs stimulate an nAChR containing the non- $\alpha$ subunit EAT-2 that clusters at the MC synapses, and eat-2 mutants exhibit the same slow pump rate observed in MC-ablated animals (Raizen and Avery 1994; McKay et al. 2004). EAT-2 stimulation initiates an increase in cytoplasmic $\mathrm{Ca}^{2+}$ concentration in the pharyngeal muscles that is mediated by the voltage-activated $\mathrm{Ca}^{2+}$ channels CCA-1 and EGL-19, leading to muscle contraction (Shtonda and Avery 2005; Steger et al. 2005; Kerr 2006). MC does not synapse on the terminal bulb, but simultaneous contraction of the corpus and the terminal bulb is mediated by electrical coupling (Starich et al. 1996). In the absence of MC or EAT-2, pumping occurs slowly, and it is believed that these contractions are generated autonomously within the muscle.

Peristalsis is stimulated by the single M4 motor neuron that extends processes through the isthmus and synapses on the muscles in the posterior half of pm5 (Albertson and Thomson 1976), and ablation of M4 eliminates peristalsis (Avery and Horvitz 1987). The receptors that respond to M4 to produce a peristalsis are unknown, but our previous studies of M4defective mutants have implicated mAChRs (Ray et al. 2008; Ramakrishnan and Okkema 2014), while other studies suggest that peptide neurotransmitters secreted from other neurons may also be involved (Song and Avery 2012). The C. elegans genome contains three genes encoding mAChRs-gar-1, gar-2, and gar-3-but only gar-3 is expressed in pharyngeal muscles (Lee et al. 2000; Steger and Avery 2004; Dittman and Kaplan 2008) and the muscarinic agonist arecoline can stimulate pumping through GAR-3 (Steger and Avery 2004).

Here, we examine the roles that nAChRs and mAChRs play in peristalsis in wild-type animals, and mutants defective in ACh synthesis or response, using both direct observation and imaging of the genetically encoded $\mathrm{Ca}^{2+}$ indicator (GECI) GCaMP3 (Tian et al. 2009). We find that mutants lacking endogenous ACh fail to pump or peristalse, indicating that pharyngeal muscle contractions are not generated autonomously. Further, treatment of these mutants with either nicotinic or muscarinic agonists stimulates both pumping and peristaltic contractions, and the response to these agonists depends on the nAChR subunit EAT-2 and the mAChR GAR-3, respectively. Surprisingly, in the absence of exogenous agonist, eat-2 mutants exhibit hyperstimulated isthmus muscle peristalses and increased isthmus muscle $\mathrm{Ca}^{2+}$ concentrations, and this hyperstimulation depends on cross talk with the GAR-3 receptor. This relationship between EAT-2 and GAR-3 affects feeding behavior throughout the life of the animal, as gar-3 mutation suppresses the slow larval growth and prolonged life span phenotypes resulting from dietary restriction in eat-2 mutants (Avery 1993a; Lakowski and Hekimi 1998). ace-3 acetylcholinesterase mutants similarly exhibit hyperstimulated isthmus peristalses that are dependent on gar-3, and we hypothesize that, in the absence of EAT-2 or ACE-3, unbound ACh spills over from the synapse and stimulates extrasynaptic GAR-3 in the isthmus muscles. 


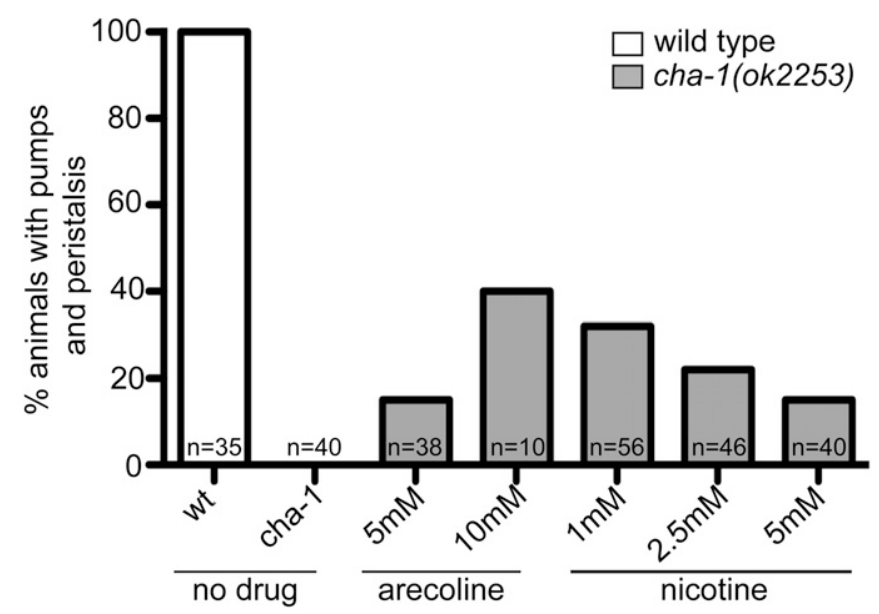

Figure 2 Muscarinic and nicotinic receptor agonists stimulate pharyngeal muscle contractions in cha-1 mutants. The percentage of wild-type (wt) or cha-1(ok2253) L1 animals exhibiting pharyngeal muscle pumping and peristalses, either untreated or treated with the indicated concentrations of arecoline or nicotine. Animals were visualized for $5 \mathrm{~min}$ each and the number of animals observed $(n)$ is indicated.

\section{Materials and Methods}

\section{Nematode handling, transformation, and strains}

C. elegans strains were grown under standard conditions (Lewis and Fleming 1995). Germline transformations were performed by microinjection using pRF4 (100 ng/ $\mu \mathrm{l}$ ) containing rol-6(su1006) as a transformation marker, and the

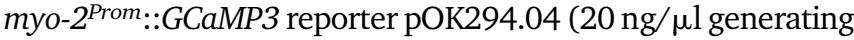
cuEx804) or the ceh-19b Prom::snb-1::gfp reporter pOK361.01(25 $\mathrm{ng} / \mu \mathrm{l}$ generating cuFx826) (Mello and Fire 1995). The extrachromosomal myo-2 $2^{\text {Prom }:: G C a M P 3 ~ t r a n s g e n e ~ c u E x 804 ~ w a s ~ c h r o-~}$ mosomally integrated by UV/trimethylpsoralen mutagenesis and outcrossed to form culs36.

The following strains were generated by the C. elegans Gene Knockout Consortium (C. elegans Deletion Mutant Consortium 2012): VC1836 cha-1(ok2253); nT1[qls51], VC657 gar-3(gk305), FX863 acr-7(tm863), RB918 acr-16(ok789), VC2661 acr-10(ok3118), RB2294 acr-6(ok3117), and VC2820 eat-2(ok3528)/mT1; +/mT1.

The following strains were constructed by others: wild-type strain N2 (Brenner 1974), RB1132 acr-14(ok1155) (Ruaud and Bessereau 2006), DA1116 eat-2(ad1116) (Raizen et al. 1995), DA465 eat-2(ad465) (Avery 1993a), PR1300 ace-3(dc2) (Combes et al. 2000), GC201 ace-2(g72); ace-1 (p1000) (Talesa et al. 1995; Culetto et al. 1999), eat-18(ad820) (Raizen et al. 1995), and UL2702 unc-119(ed3); leIs2702(ceh-19b Prom::gfp, unc-119(+)) (Feng and Hope 2013).

The following strains were constructed in this study: OK1020 culs36[myo-2 ${ }^{\text {Prom }:: G C a M P 3], ~ O K 1062 ~ g a r-3(g k 305) ; ~}$ culs36, OK1063 eat-2(ok3528); culs36, OK1064 eat-2(ok3528); gar-3(gk305), OK1075 eat-2(ok3528); gar-3(gk305); culs36, OK1023 eat-2(ok3528), OK1081 ace-3(dc2); gar-3(gk305),

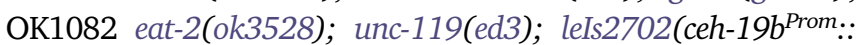

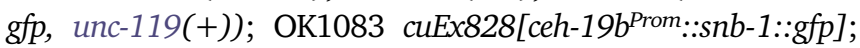
and OK1084 eat-2(ok3528); cuEx828.

\section{General methods for nucleic acid manipulations and plasmid construction}

Standard methods were used to manipulate all DNAs (Ausubel 1990) and plasmid sequences are available from the authors. The myo- 2 promoter from plasmid pPD96.48 was cloned into a HindIII- and MscI-digested str-2::GCaMP3 plasmid (Chalasani et al. 2007) to generate pOK294.04. The ceh-19b promoter was amplified from N2 genomic DNA using primers PO1452 (5'-CGAGCATGCGAAAAACAGGAAAGTC TCG-3') and PO1453 (5'-GACCCGGGATGTAGAGTTGAGAA GTTGCCA-3'), digested with SphI and XmaI, and inserted into SphI- and XmaI-digested ser-7b ${ }^{\text {Prom }}:: s n b-1:: g f p$ plasmid pOK219.08 to generate the ceh-19b Prom::snb-1::gfp plasmid pOK361.01 (Ray et al. 2008).

\section{Genotyping}

Individual animals were genotyped for eat-2(ok3528), gar$3(g k 305)$, and ace-3(dc2) alleles by PCR (Beaster-Jones and Okkema 2004). Primers for genotyping eat-2(ok3528) were PO1423 (5'-TGCGTGGTAGAGGGATAGTG-3'), PO1424 (5' TCTCGACGAGACCTACGTTG-3'), and PO1425 (5'-ACAGCT ACAGTACCTCGCAC-3'). Primers for genotyping gar-3(gk305) were PO1426 (5'-TAATAGGTTCGGCCCAGAGC-3'), PO1427 (5'-GTGATCGTTTGCTGGGAAGC-3'), and PO1428 (5' -CGAA GCTCAGAATGTCAGTAACG-3'). Primers for genotyping ace3(dc2) were P01435 (5'-CAAGGATACAGAGTACACGGCA- $\left.3^{\prime}\right)$, PO1436 (5' -CAAGCCCGCAAATTGAACTGA-3'), and P01437 (5'-GCAAGTGGCAAGCGAGAATA-3').

\section{Analysis of feeding behavior and drug studies}

To analyze pharyngeal muscle contractions, L1 larvae hatched in the absence of food were suspended in $5 \mu \mathrm{l}$ of M9 buffer containing OP50 and imaged on a $2 \%$ agarose pad under a coverslip. For drug treatments, either arecoline [catalog number (Cat\#) CAS: 300-08-3; Acros Organics] or nicotine (Cat\# N5260-25G; Sigma [Sigma Chemical], St. Louis, MO) was included, and animals were treated for $15 \mathrm{~min}$ prior to adding a coverslip. Individual N2 or mutant animals that pumped were recorded at 25 frames/sec for $1 \mathrm{~min}$ (cha-1 mutants were recorded for $5 \mathrm{~min}$ ) using a Zeiss ([Carl Zeiss], Thornwood, NY) AxioImager microscope with an MRm camera and ZEN Software. For each genotype and drug treatment, the feeding behavior was analyzed in at least five animals ( $\sim 500$ pumps/per animal). Time-lapse images were exported and processed using Fiji/ImageJ (Schindelin et al. 2012) to generate time-lapse movies. Acquisition times were exported from ZEN and quantifications were performed using Microsoft Excel.

\section{Calcium imaging and microscopy}

Young adult animals were incubated in $5 \mu \mathrm{l}$ of $20 \mathrm{mM}$ serotonin (Cat\# H7752-5G; Sigma) for 10 min on a $2 \%$ agarose pad, and then immobilized using $1.5 \mu \mathrm{l}$ polystyrene $0.10 \mu \mathrm{m}$ microspheres and a coverslip (Cat\# 00876; Polysciences, Warrington, PA). GCaMP3 imaging was performed on a Zeiss AxioImager microscope using a Q-Imaging Rolera EM-C ${ }^{2}$ 
Table 1 Quantification of pharyngeal muscle contractions

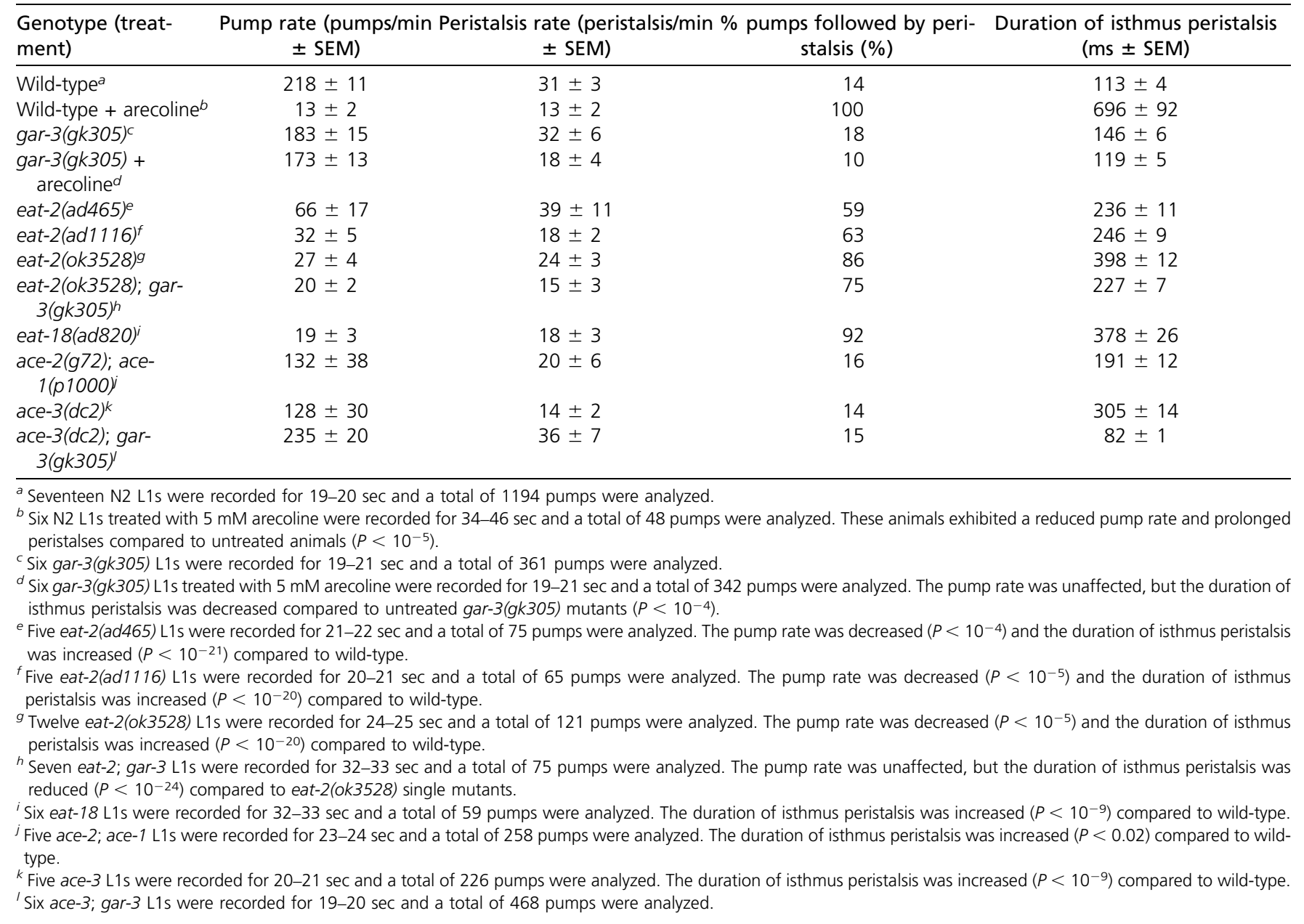

EMCCD camera. Time-lapse movies were captured at 25-30 frames/sec using ZEN software and 14-bit TIFF images were exported. Animals pumping with a frequency of $<100$ pumps/min were analyzed. For quantification, the pharynx was straightened with CellProfiler (Kamentsky et al. 2011) and aligned using the StackReg Fiji plugin (Thevenaz et al. 1998). The isthmus was cropped, and two regions of interest (ROI) were drawn as 10 pixel $(2.54 \mu \mathrm{m})$-wide lines in the center and posterior isthmus. Fluorescence measurements were analyzed using custom Matlab scripts or using Microsoft Excel. Total fluorescence was measured within the ROI and normalized for each GCaMP3 peak using the formula: normalized $\Delta \mathrm{F}=(\mathrm{Fmax}-\mathrm{Fmin}) / \mathrm{Fmin} * 100 \%$ (where Fmax is the maximum fluorescence of the GCaMP3 peak and Fmin is the minimum fluorescence immediately before the GCaMP3 increase). Peak duration was quantified as a width of GCaMP3 fluorescence at half-peak height. Rise time was quantified as the time that it took for each fluorescence peak to reach its maximum. A Student's $t$-test was used to compare GCaMP3 fluorescence measurements between different genotypes and boxplots were generated using the Matlab boxplot command. Peak delay was calculated as the time between the maximum increase in GCaMP3 fluorescence in the center and posterior isthmus using the Matlab diff command. Average baseline fluorescence levels in the entire pharyngeal isthmus were measured in a representative image during an interpump period acquired at a time point early in time-lapse image sets of each animal using Fiji/ImageJ.

GFP and DIC images were captured using a Zeiss Axiocam MRm and Zeiss Zen software. To characterize ceh-19b ${ }^{\text {Prom }:: g f p}$ expression, Z-series images were collected. Maximum intensity Z projections were produced using Fiji/ImageJ (Schindelin et al. 2012). ceh-19b ${ }^{\text {Prom }:: s n b-1:: g f p ~ e x p r e s s i o n ~ w a s ~ f a i n t, ~ a n d ~}$ Z-series were collected in adult animals immobilized in $10 \mathrm{mM}$ $\mathrm{NaN}_{3}$ and false colored in Fiji/ImageJ using the Rainbow RGB look up table.

\section{Growth assay}

Adult hermaphrodites were allowed to lay eggs for $8 \mathrm{hr}$ at $25^{\circ}$, and embryos were transferred to freshly seeded plates and incubated at $20^{\circ}$ for $16 \mathrm{hr}$. Unhatched embryos were counted and hatched L1s were allowed to grow for an additional 3 days. Adult animals were counted and removed on day 5. eat-2(ok3528) and eat-2(ok3528); gar-3(gk305) 


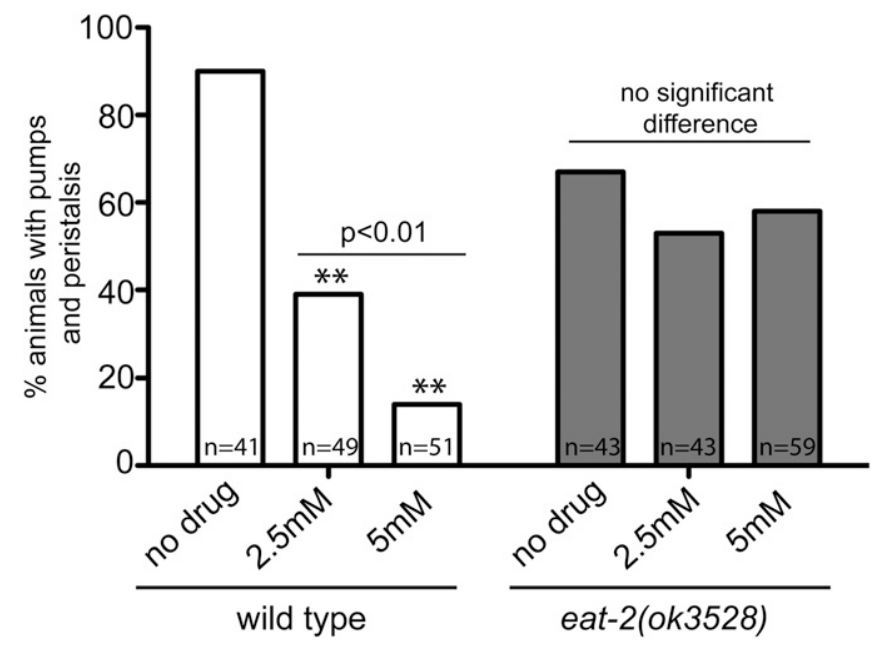

Figure 3 eat-2 mutants are insensitive to exogenous nicotine. Percentage of wild-type or eat-2(ok3528) L1 animals exhibiting pharyngeal muscle pumping and peristalses, either untreated or treated with the indicated concentrations of nicotine. $* *$ indicates significantly different from untreated animals $(P<0.0001)$, and the bar indicates significant difference between wild-type animals treated with increasing concentrations of nicotine. Animals were visualized for 5 min each and the number of animals observed $(n)$ is indicated.

animals were allowed to grow for an additional day to quantify slow-growing adults. For each genotype, two plates were set up with 50 embryos each.

\section{Life span assay}

Life span assays were performed according to a previously published protocol (Sutphin and Kaeberlein 2009). Assays were performed starting with $30 \mathrm{~L} 4$ worms fed with UV-killed OP50 Escherichia coli at $20^{\circ}$, and dead or missing animals were replaced by animals of the same age. For each experiment, triplicate plates of each genotype were assayed, and representative results of three or more experiment are shown. Kaplan-Meier survival analysis was used to generate survival plots and calculate median life span, and for statistical analysis (GraphPad Prism 5).

\section{Data availability}

Strains and plasmids are available upon request. Supplemental Material, Figure S1 contains measurements of baseline GCaMP3 fluorescence intensity in wild-type and mutant strains. Figure S2 contains tracings of GCaMP3 fluorescence intensity from representative wild-type and mutant animals. Movies S1-S9 include time-lapse images of wild-type and mutant $C$. elegans. Table $S 1$ contains measurements of pharyngeal muscle contractions in acr mutant C. elegans. Tables S2-S52 contain raw data of pharyngeal muscle contractions (Tables S2-S13) and GCaMP3 fluorescence intensities (Tables S14 S52) derived from time-lapse images. Supplemental material available at Figshare: https://doi.org/10.25386/genetics. 6160355.

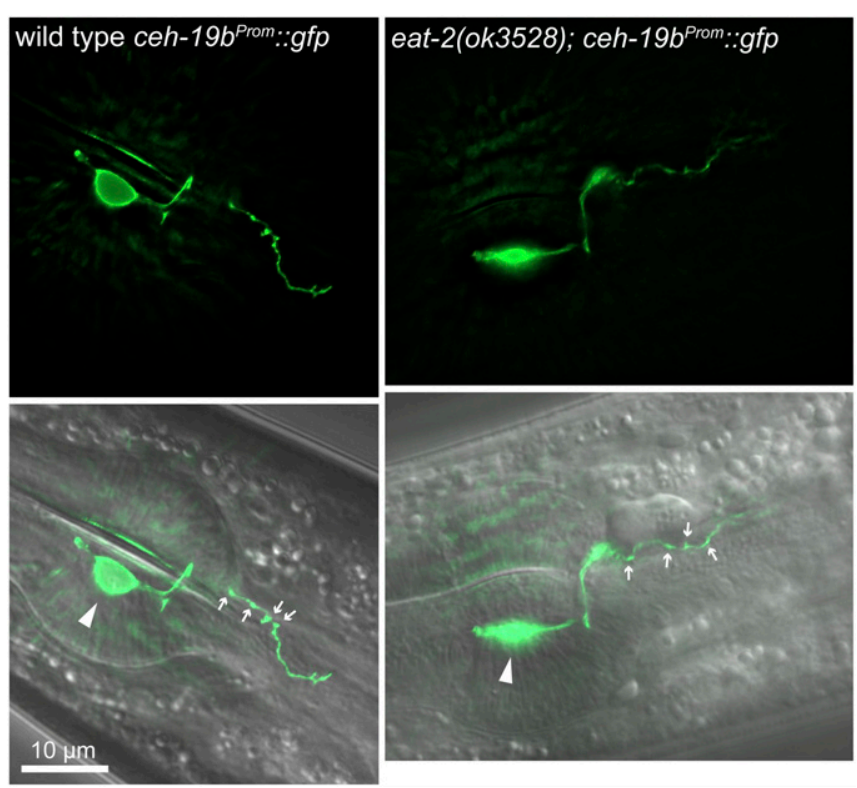

Figure $4 \mathrm{MC}$ neuron morphology in wild-type and eat-2 mutant adults. Fluorescence (top), and merged fluorescence and DIC images of adult animals of the indicated genotypes expressing ceh-19bProm::gfp in the $M C$ neuron. Anterior is left, and the metacorpus and anterior isthmus are shown. The MC cell body (arrowhead) and varicosities in the MC process (small arrows) are indicated. Fluorescence images are maximal intensity Z-projections of images through one $\mathrm{MC}$ cell and process.

\section{Results}

\section{Pumping and isthmus peristalsis can be stimulated through both nicotinic and muscarinic receptors}

We are interested in the signaling mechanisms that produce pharyngeal muscle contractions and productive feeding. M4 and the MCs are cholinergic motor neurons that express choline acetyltransferase encoded by cha-1 (Raizen et al. 1995; Ramakrishnan and Okkema 2014; Pereira et al. 2015), and strong cha-1 mutants have reduced pharyngeal contractions and arrest as severely uncoordinated L1s (Rand 1989; Avery and Horvitz 1990). Previous studies have shown that stimulation of nAChRs with nicotine or mAChRs with arecoline could induce pharyngeal muscle contractions, but these studies did not specifically examine peristalses in the absence of endogenous ACh (Avery and Horvitz 1990; Raizen et al. 1995; Ramakrishnan and Okkema 2014).

To extend this work, we examined the effect of nicotine or arecoline treatment on cha-1(ok2253) mutant L1 animals. cha-1(ok2253) is a previously uncharacterized null allele containing a 1.7-kb deletion that eliminates the catalytic histidine (His341) required for ACh synthesis, and homozygous mutants arrest as paralyzed L1s with a coiled appearance (C. elegans Deletion Mutant Consortium 2012). Untreated cha1 (ok2253) homozygotes completely lacked pharyngeal muscle contractions, but mutant animals treated with either nicotine or arecoline exhibited both pumping and peristalsis (Figure 2 and Movies S1-S4). These results demonstrate that ACh is necessary for pumping and peristalsis, indicating that 

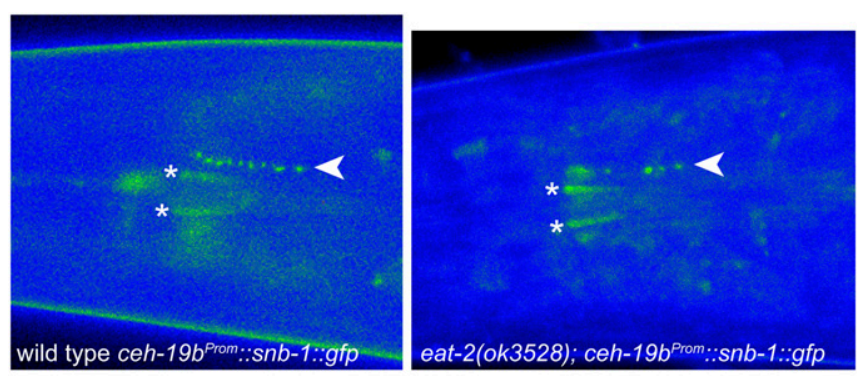

\section{EAT-2 and GAR-3 mediate the response to agonists}

The results above indicate that activation of either mAChRs or nAChRs can stimulate pumping and peristaltic contractions in the pharynx. To identify the receptors involved in these responses, we asked if mutants affecting receptors expressed in the pharyngeal muscles become insensitive to agonists.

To examine the role that $\mathrm{mAChR}$ signaling plays in pharyngeal muscle contractions, we characterized these contractions in gar-3(gk305) null mutants (Liu et al. 2007). gar-3(gk305) mutants are viable, look healthy, and grow normally, and pharyngeal muscle contractions in gar-3(gk305) L1s were similar to those in wild-type worms (Table 1). Neither the rate of pumping, nor the frequency or duration of peristalsis, was significantly different between wild-type and gar-3(gk305) animals. In comparison, gar-3(gk305) animals were almost completely insensitive to exogenously applied arecoline (Table 1). Wild-type animals treated with arecoline exhibited a strongly reduced pump rate, and each of these pumps was followed by a peristalsis that was prolonged compared to untreated animals. In contrast, gar-3 (gk305) mutants were largely unaffected by arecoline. The pump rate was unaffected in gar-3(gk305) and, although these animals did exhibit a small decrease in the duration of peristaltic contractions when treated with arecoline, we note that this change is the opposite of the prolonged peristalses observed in arecolinetreated wild-type animals. Thus, GAR-3 mediates the response to arecoline, but loss of this receptor does not have a strong

Figure $5 \mathrm{MC}$ neuron synapses in wild-type and eat-2 mutant adults. Fluorescence (top), DIC (middle), and merged images (bottom) of the adult animals of the indicated genotypes expressing ceh-196 Prom::snb$1:: \mathrm{gfp}$ in the MC neuron. Anterior is left, and the metacorpus and anterior isthmus are shown. Synaptic vesicle clusters marking en passant synapses in the axon of one MC neuron are marked in the fluorescence and merged images (arrowheads). Nonspecific autofluorescence at the edges of the pharyngeal lumen are marked in the fluorescence and DIC images (asterisks). Fluorescence images are false colored using the look-up table at the bottom right.

pharyngeal muscles do not have autonomous contractile activity. Further, activation of either nAChRs or mAChRs can stimulate these contractions. Notably, we observed occasional animals treated with either agonist that exhibited peristaltic contractions in the isthmus without a preceding pump (2/10 animals treated with $10 \mathrm{mM}$ arecoline and 2/40 animals treated with $5 \mathrm{mM}$ nicotine), consistent with our previous observations that peristalsis can be uncoupled from pumping by directly stimulating receptors in the isthmus muscles (Ramakrishnan and Okkema 2014). In addition, while higher arecoline concentrations increased the percentage of animals exhibiting pharyngeal muscle contractions, higher nicotine concentration progressively decreased the percentage of animals exhibiting pharyngeal muscle contractions. This decrease may result from hyperstimulation of nAChRs, as has been previously reported (Avery and Horvitz 1990), although we did not observe the tetanic contractions previously observed in animals treated with nicotine.

effect on pharyngeal muscle contractions in the absence of this drug.

We then focused on eat-2, which encodes the only nAChR subunit known to function in the pharyngeal muscles (Raizen et al. 1995; McKay et al. 2004). To test if eat-2 is necessary to respond to nicotine, we compared wild-type animals and eat2(ok3528) mutants treated with nicotine at the L1 stage. Like cha-1 mutants, wild-type animals treated with increasing concentrations of nicotine exhibited a dose-dependent decrease in pharyngeal muscle contractions (Figure 3), but eat-2 animals were not significantly affected by this treatment. Thus, the pharyngeal muscle response to nicotine depends on EAT-2-containing nAChRs.

\section{eat-2 mutants exhibit prolonged pumps and peristalses}

To understand how EAT-2 affects muscle contractions, we examined three strong eat-2 mutants at the L1 stage. eat2(ad465) and eat-2(ad1116) are point mutations introducing an early stop codon and affecting a splice site, respectively (McKay et al. 2004) (WormBase WBVar00000089), while eat-2(ok3528) contains a 614-bp deletion predicted to cause a frameshift mutation upstream of the transmembrane domains (C. elegans Deletion Mutant Consortium 2012). As expected, the rate of pumping in all three mutants was significantly reduced compared to in wild-type worms (Table 1) (Avery 1993a; Raizen et al. 1995). However, the duration of peristaltic contractions in the posterior isthmus was unexpectedly increased up to nearly fourfold, with relaxation of 


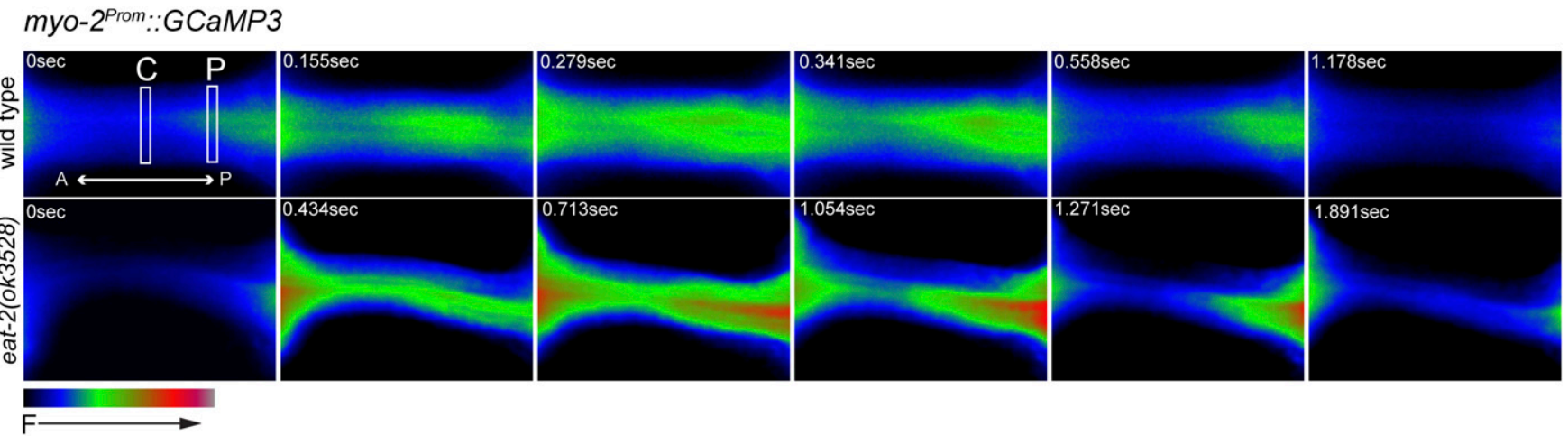

Figure 6 Dynamic changes in $\mathrm{Ca}^{2+}$ levels in the isthmus muscles. Time-lapse fluorescence images of the pharyngeal isthmus of a wild-type (top) or eat2(ok3528) (bottom) adult expressing the genetically encoded Ca ${ }^{2+}$ indicator GCaMP3 in the pharyngeal muscles. Images are false colored as indicated at the lower left, and one pump and peristalsis are shown. Boxes indicate the regions where fluorescence levels were quantified in the center (C) and posterior $(P)$ isthmus. The amounts of time after fluorescence begins to increase are indicated, and the frames indicating maximum fluorescence in wildtype and eat-2(ok3528) are shown at 0.279 and $0.713 \mathrm{sec}$, respectively.

the posterior region of the isthmus muscles particularly delayed (Movie S5). The percent of pumps that were followed by a peristalsis was also increased in eat-2 mutants, but as the pump rate was decreased in these animals, the absolute rate of peristalsis was similar in wild-type animals and eat-2 mutants. Notably, eat-2(ok3528) mutants exhibited the strongest phenotypes, which, together with a molecular lesion in this allele, suggests that eat-2(ok3528) is a null allele.

We next examined MC in eat-2(ok3528) mutants using a ceh-19b 19 rom::gfp reporter (Feng and Hope 2013), and found that MC morphology is similar in wild-type and eat2(ok3528) adults (Figure 4). The MC cell bodies were located in the anterior bulb of the pharynx and these cells extended processes posteriorly into the pharyngeal isthmus. Varicosities in these processes that mark synaptic sites were observed in both strains at the junction between the pm4 and pm5 muscles, and in the anterior region of the isthmus. We then looked specifically at the locations of synapses in MC using a snb-1::gfp fusion expressed using the ceh-19b promoter. SNB-1::GFP is a functional synaptobrevin that marks synaptic vesicle clusters in presynaptic cells (Nonet 1999). In both wild-type adults and eat-2(ok3528) mutants, synapses were visible in the MC process beginning near the junction of the pm4 and pm5 muscle cells, and extending into the anterior region of the isthmus (Figure 5). eat-2(ok3528) mutants exhibited a small but not statistically significant decrease in the number of vesicle clusters compared to wild-type (wildtype $7.0 \pm 2.40$ clusters, $n=8$; eat-2(ok3528) $4.9 \pm 1.0 ; P=$ $0.09, n=13)$. Thus, the prolonged peristalses in eat- $2 \mathrm{mu}-$ tants do not result from morphological or synaptic defects in the MCs. Notably, using both of these reporters, we observed the MC axon extending into the anterior isthmus further than has been previously reported (Albertson and Thomson 1976) and forming synapses directly on the pm5 muscles.

To corroborate our observations in eat-2 mutants, we also examined pharyngeal muscle contractions in eat-18 mutants. eat-18 encodes a novel transmembrane protein required for the function of EAT-2 and other nAChRs in the pharynx, and eat-18 mutants share the reduced pump rate with eat-2 mutants (Raizen et al. 1995; McKay et al. 2004). We found that eat-18(ad820) animals also exhibit prolonged peristalses with almost all pumps followed by peristalses (Table 1). In contrast, mutants affecting several other nAChR subunits reported to be expressed in the pharyngeal muscles, including acr-6 (ok3117), acr-7(tm863), acr-10 (ok3118), acr-14(ok1155), and acr-16(ok789) (Saur et al. 2013), did not exhibit significant changes in pumping frequency, peristalsis frequency, or the duration of peristalsis (Table S1).

Taken together, these results indicate that loss of the EAT-2 nAChR subunit results in slow pumping and prolonged peristalses that occur after nearly every pump. Paradoxically, the eat-2 mutant defects in peristalsis are opposite to those in pumping, indicating that, while wild-type EAT-2 stimulates rapid pumping, it also limits the duration of peristaltic contractions in the isthmus muscles.

\section{gar-3 mutation suppresses the peristalsis defects in eat-2 mutants}

Pharyngeal muscle contractions in eat-2 mutants are strikingly similar to those of wild-type animals treated with arecoline (Table 1), suggesting that some of the eat-2 mutant phenotypes are related to mAChR signaling. Since GAR-3 is the mAChR responding to arecoline in the pharynx, we examined pharyngeal muscle contractions in eat-2(ok3528); gar-3(gk305) double mutants.

We found that gar-3 mutation partially suppressed the prolonged peristalses observed in eat-2 single mutants (Table 1 ). The duration of peristalses in eat-2(ok3528); gar-3(gk305) double mutants was strongly reduced compared to eat2(ok3528) single mutants, although they were still longer than those in wild-type animals or gar-3(gk305) single mutants. In contrast, the reduced pumping frequency and increased frequency of peristalsis observed in eat-2(ok3528) single mutants was not suppressed in eat-2(ok3528); gar3 (gk305). Thus, eat-2 loss-of-function affects pumping and 

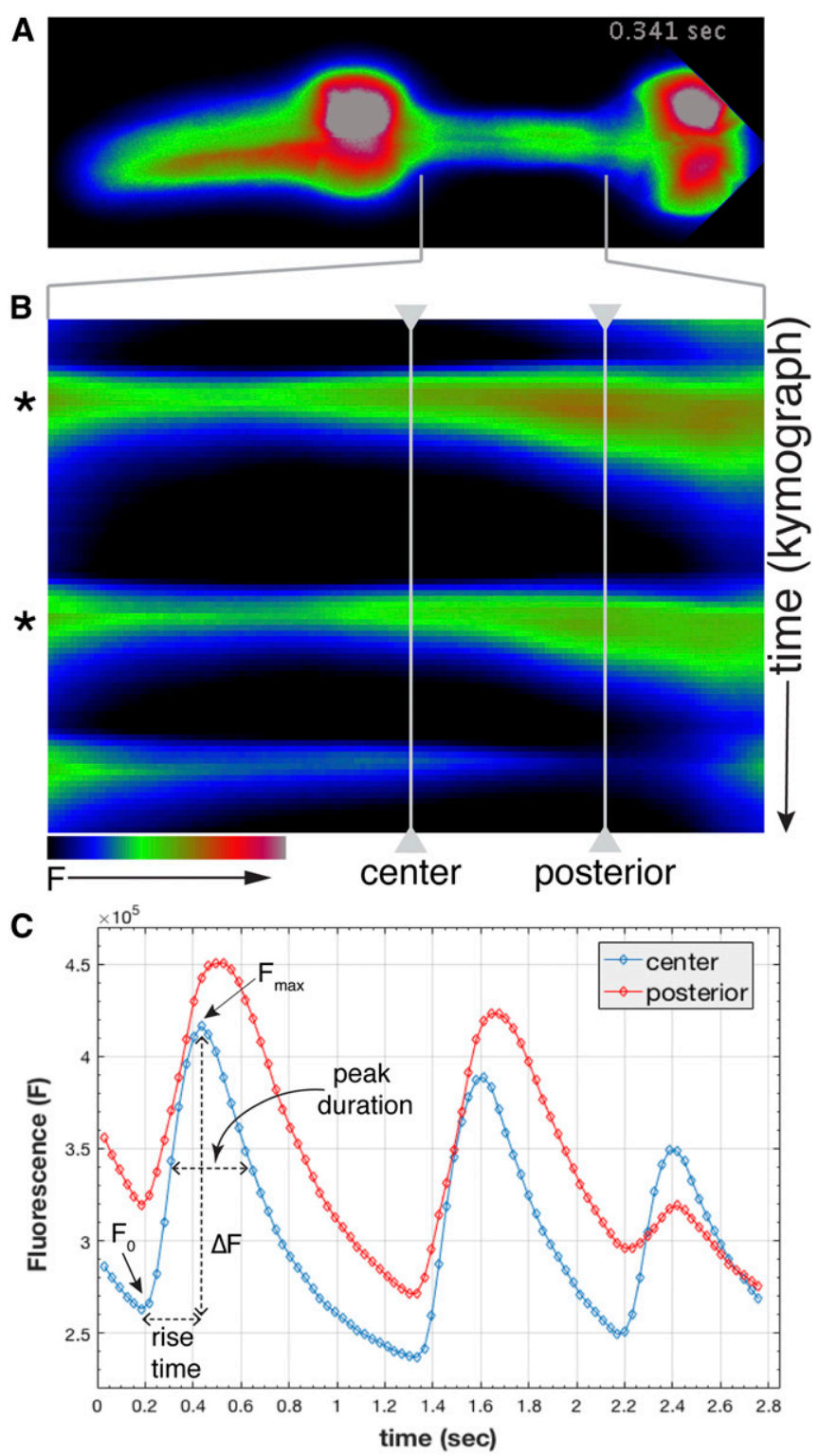

Figure 7 Characterization of GCaMP3 fluorescence in the pharyngeal isthmus. (A) False-colored fluorescence image of a wild-type animal expressing GCaMP3 in the pharyngeal muscles (anterior is left). (B) Kymograph of maximum GCaMP3 fluorescence intensity in the isthmus region [indicated by brackets in (A and $B)$ ]. Two pumps with peristalsis marked with asterisks are followed by a pump without a peristalsis. (C) Fluorescence levels (F) in the center and posterior isthmus plotted vs. time (seconds) for the contractions in (B). Time points for time-lapse imaging (circles) and example measurements for $\Delta \mathrm{F}$, peak duration, and rise time are indicated

peristalsis by different mechanisms, and only the prolonged peristalsis phenotype depends on cross talk with the GAR-3 receptor.

\section{$\mathrm{Ca}^{2+}$ transients in the pharyngeal isthmus parallel the contractile phenotypes in eat-2 and gar-3 mutants}

To characterize isthmus muscle excitation in wild-type animals and mutants, we constructed strains expressing the GECI GCaMP3 using the pharyngeal muscle-specific myo-2 promoter
(Okkema et al. 1993; Tian et al. 2009). GCaMP3 fluorescence increases in muscle cells as cytoplasmic $\mathrm{Ca}^{2+}$ concentrations increase during excitation contraction coupling [reviewed Bers (2002)]. Similar to previous analyses of GECIs in the pharynx, we characterized adult animals treated with serotonin to stimulate pharyngeal muscle contractions and focused on animals pumping slowly to resolve individual excitation events ( $<100 \mathrm{pumps} / \mathrm{min} ;<1.67 \mathrm{~Hz}$ ) (Shimozono et al. 2004; Kerr 2006). Baseline fluorescence levels in the isthmus muscles were similar in the strains examined (Figure S1) and representative traces of time-lapse fluorescence are shown in Figure S2.

We observed changes in $\mathrm{Ca}^{2+}$ concentration in the isthmus muscles that were very dynamic (Figure 6 and Movie S6). As reported in previous studies, we observed that wild-type animals displayed an increase in $\mathrm{Ca}^{2+}$ throughout the central isthmus during pumping, followed by a longer and delayed increase in $\mathrm{Ca}^{2+}$ in the posterior isthmus during peristalsis (Figure 7, A and B and Movie S6) (Shimozono et al. 2004). We found that this $\mathrm{Ca}^{2+}$ signal during peristalsis exhibited a wave-like increase and decrease that traveled in an anterior to posterior direction through the posterior isthmus, and resembled the progression of the open pharyngeal lumen during peristalsis (Movie S7). While $\mathrm{Ca}^{2+}$ waves have not been previously described in the C. elegans pharynx (Shimozono et al. 2004), similar waves of $\mathrm{Ca}^{2+}$ can be observed in individual cardiac muscle cells, which are generated by progressive release of $\mathrm{Ca}^{2+}$ released from adjacent sites in the sarcoplasmic reticulum [reviewed in Stuyvers et al. (2000)]. This mechanism may also underlie the wave-like contraction of pm5 during peristalsis.

To compare $\mathrm{Ca}^{2+}$ transients in the center and posterior isthmus in wild-type animals to those in various mutants, we quantified for each pump and peristalsis the normalized change in fluorescence $\left(\Delta \mathrm{F} / \mathrm{F}_{\mathrm{o}}\right)$, the width of each peak at $50 \%$ maximum fluorescence (peak duration), and the time that it took for fluorescence to reach its maximum (rise time) (Figure 7C and Table 2). In addition, we calculated the time delay between the maximum increase in GCaMP3 fluorescence in the center and posterior isthmus (peak delay).

We found changes in GCaMP3 fluorescence in the pharyngeal isthmus that paralleled many of the defects we observed in peristaltic contractions in various mutants (Figure 8 and Table 2). eat-2(ok3528) mutants, which have prolonged peristalses, also exhibited significantly increased $\Delta \mathrm{F} / \mathrm{F}_{\mathrm{o}}$, peak duration, and rise time, in both the center and posterior isthmus. In addition, the peak delay was increased twofold (Movies S8 and S9). In comparison, gar-3(gk305) mutants, which have normal duration of peristalses, exhibited $\Delta \mathrm{F} / \mathrm{F}_{\mathrm{o}}$ and rise times that were similar to those of wild-type animals, with a small decrease in $\Delta F / F_{o}$ only in the posterior isthmus, as well as a small decrease in peak duration in both the center and posterior isthmus. However, the most striking change in these animals was a strongly decreased peak delay between the center and posterior 
Table 2 Quantification of GCaMP3 fluorescence dynamics

\begin{tabular}{|c|c|c|c|c|c|}
\hline Genotype & Isthmus position & Normalized $\Delta \mathrm{F}$ & Peak duration ( $\mathrm{ms} \pm \mathrm{SEM})$ & Rise time (ms \pm SEM) & Peak delay $(\mathrm{ms} \pm \mathrm{SEM})$ \\
\hline \multirow[t]{2}{*}{ wild type ${ }^{a}$} & Center & $52 \pm 2$ & $329 \pm 7$ & $256 \pm 5$ & \\
\hline & Posterior & $40 \pm 2$ & $376 \pm 10$ & $279 \pm 6$ & $33 \pm 4$ \\
\hline \multirow[t]{2}{*}{ eat-2(ok3528)b } & Center & $78 \pm 3$ & $598 \pm 30$ & $432 \pm 9$ & \\
\hline & Posterior & $63 \pm 3$ & $666 \pm 39$ & $426 \pm 12$ & $67 \pm 7$ \\
\hline \multirow[t]{2}{*}{ gar-3(gk305)c } & Center & $51 \pm 3$ & $310 \pm 11$ & $258 \pm 8$ & \\
\hline & Posterior & $34 \pm 2$ & $345 \pm 14$ & $281 \pm 10$ & $7 \pm 3$ \\
\hline \multirow[t]{2}{*}{ eat-2; gar-3 $3^{d}$} & Center & $57 \pm 2$ & $414 \pm 12$ & $315 \pm 6$ & \\
\hline & Posterior & $36 \pm 2$ & $485 \pm 17$ & $346 \pm 8$ & $26 \pm 4$ \\
\hline
\end{tabular}

${ }^{a}$ Fifteen wild-type OK1020 young adults were recorded and an average of 130 pumps were analyzed.

${ }^{b}$ Six eat-2(ok3528) young adults were recorded and an average 56 pumps were analyzed. These animals exhibited increased normalized $\Delta \mathrm{F}$, peak duration, and rise time in both the center and posterior isthmus $\left(P<10^{-7}\right)$, and an increased peak delay $\left(P<10^{-4}\right)$ compared to wild-type.

${ }^{c}$ Five gar-3(gk305) young adults were recorded and an average of 31 pumps were analyzed. These animals exhibited a decreased peak delay $\left(P<10^{-5}\right)$ compared to wildtype.

${ }^{d} 12$ eat-2; gar-3 young adults were recorded and an average of 94 pumps were analyzed. These animals exhibited decreased normalized $\Delta \mathrm{F}$, peak duration, rise time, and peak delay compared to eat-2(ok3528) single mutants $\left(P<10^{-5}\right)$.

isthmus. Finally, eat-2(ok3528); gar-3(gk305) double mutants, which suppress the prolonged peristalses observed in eat-2(ok3528) single mutants, exhibited decreases in $\Delta \mathrm{F} /$ $\mathrm{F}_{\mathrm{o}}$, peak duration, rise time, and peak delay compared to eat-2(ok3528) single mutants, although only $\Delta \mathrm{F} / \mathrm{F}_{\mathrm{o}}$ was reduced to wild-type levels. Taken together, these results show that the increases in level, duration, and rise time of $\mathrm{Ca}^{2+}$, in both the center and posterior isthmus, best correlate with the duration of peristaltic contractions. Loss of eat-2 increases these $\mathrm{Ca}^{2+}$ signals and produces prolonged peristalses that depend on wild-type gar-3.

\section{ace-3 mutants exhibit prolonged peristalses that depend on GAR-3}

It is surprising that eat-2 loss leads to a reduced pump rate, while at the same time hyperstimulating peristalsis and increasing the cytoplasmic $\mathrm{Ca}^{2+}$ concentration in the isthmus muscles. One hypothesis for these unexpected effects is that, in eat-2 mutants, ACh released from MC spills over from the synapses and stimulates GAR-3 receptors located in the isthmus muscles. To examine this hypothesis, we characterized pharyngeal muscle contractions in ace mutants defective in acetylcholinesterases that normally hydrolyze ACh and have increased unbound ACh in the synapse (Johnson et al. 1988; Combes et al. 2000). ace-3(dc2) mutants exhibited significantly prolonged peristalses, which were similar to those of eat-2 mutants (Table 1). In comparison, ace-2(g72); ace-1 (p1000) double mutants exhibited only mildly prolonged peristalses. Both of these strains also exhibited a small decrease in the pump rate, but this was not significantly different from wild-type, indicating that MC can effectively stimulate pumping in ace mutants.

To ask if the prolonged peristalses were suppressed by gar-3 mutation, we examined ace-3(dc2); gar-3(gk305) double mutants and found that these animals exhibited peristalses that were similar to wild-type (Table 1). Thus, the prolonged peristalses in ace-3 mutants depend on cross talk with the wildtype GAR-3 receptor, which is similar to what we observed in eat-2 mutants.

\section{gar-3 suppresses the slow growth and extended life span of eat-2 mutants}

As gar-3 mutation suppresses the peristalsis defects in eat-2 mutants, we wanted to determine if this mutation also affects the persistent feeding defects in eat-2 mutants. eat-2 mutants exhibit slow larval growth and a prolonged adult life span, which result from dietary restriction (Avery 1993a; Lakowski and Hekimi 1998). We found that gar-3 mutation at least partially suppressed both of these phenotypes.

While nearly all wild-type animals and gar-3 (gk305) single mutants reached adulthood after 5 days at $20^{\circ}$, eat- 2 mutants grew much more slowly and, even after 6 days, only $30 \%$ had reached adulthood (Figure 9A). eat-2(ok3528); gar-3(gk305) double mutants also grew slower than wild-type and gar3 (gk305), but they grew faster than eat-2(ok3528) single mutants and $100 \%$ of these animals reached adulthood within 6 days.

Likewise, eat-2 mutants have previously been shown to exhibit a longer adult life span than wild-type animals (Lakowski and Hekimi 1998), and we found that both eat2(ad1116) and eat-2(ok3528) mutants exhibited significantly extended life spans (median adult survival 22 and 20 days, respectively) (Figure 9B). In comparison, the life span of gar-3 (gk305); eat-2(ok3528) double mutants was indistinguishable from that of wild-type animals and gar-3(gk305) single mutants [median adult survival: N2 (13 days), gar3(gk305) (12.5 days), and gar-3(gk305); eat-2(ok3528) (13 days) ].

Thus, gar-3(gk305) suppresses both the slow larval growth and extended life span phenotypes of eat-2(ok3528) mutants, and we suggest that this suppression is the result of improved isthmus peristalsis and feeding.

\section{Discussion}

In this work, we show that cross talk between the nAChR EAT-2 and the mAChR GAR-3 affects peristaltic muscle contractions in the isthmus of the C. elegans pharynx. Under normal circumstances, GAR-3 has a relatively minor role in 
A GCaMP3 fluorescence
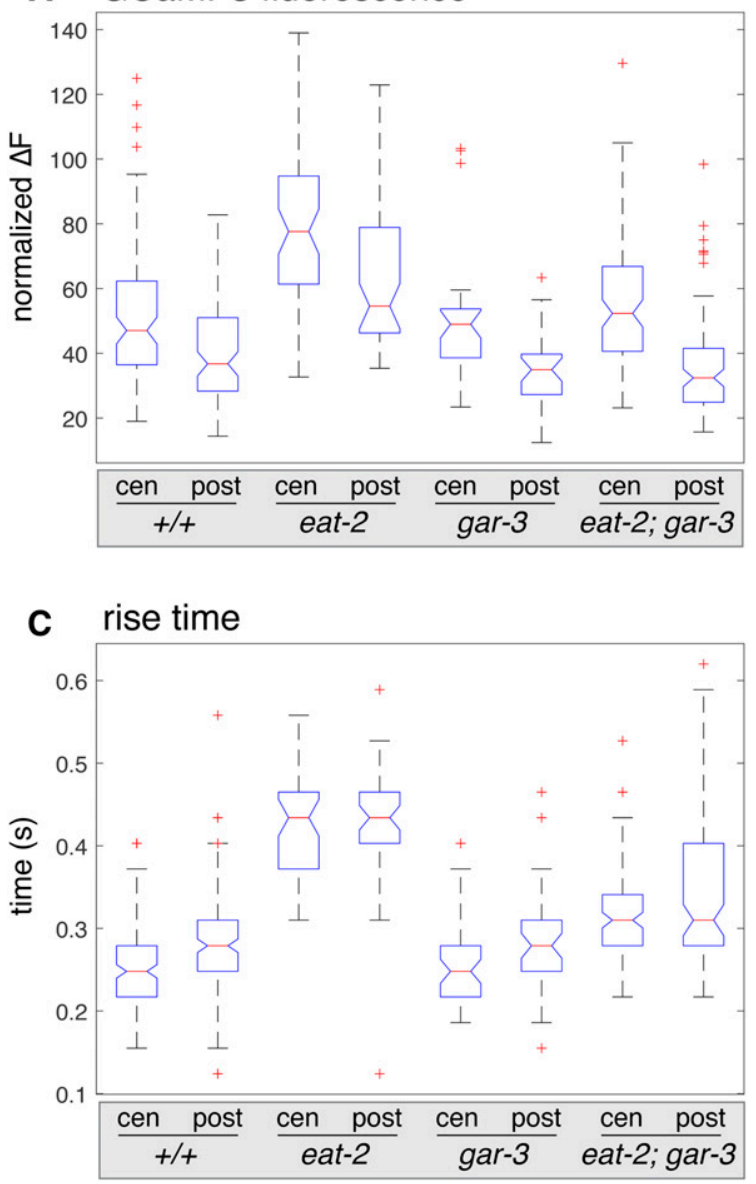
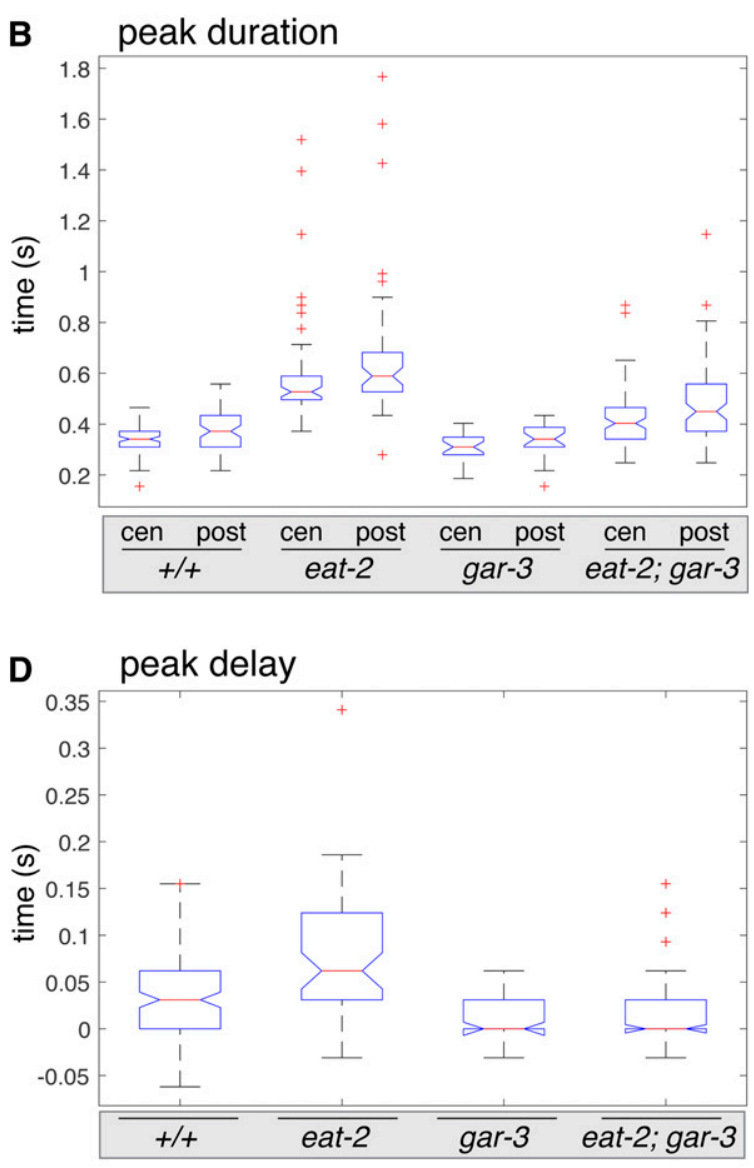

Figure 8 Quantification of GCaMP3 fluorescence in wild-type animals and mutants. Box and whisker plots comparing values measured from time-lapse imaging of GCaMP3 fluorescence during pumps followed by peristalses: $(A)$ normalized GCaMP3 fluorescence levels $\left(\Delta F / F_{0}\right),(B)$ peak duration, $(C)$ peak rise time, and (D) peak delay between the center and posterior isthmus. Genotypes and measurements in the center (cen) and posterior (post) isthmus are indicated. The central bars (red) denote the median values with notches indicating the $95 \%$ C.I.s for the median, the boxes indicates the interquartile ranges (IQRs, 25th to 75th percentile), and the whiskers indicate values within 1.5 times the IQR. Suspected outlier values are indicated as red "+."

these contractions, but in eat-2 mutants, GAR-3 can stimulate increased cytoplasmic $\mathrm{Ca}^{2+}$ levels and peristalses that are prolonged compared to those of wild-type animals. We suggest that this GAR-3-dependent stimulation of peristalsis results from ACh spillover from synapses between MC and the pharyngeal muscles in eat-2 mutants, and, consistent with this suggestion, acetylcholinesterase mutants similarly exhibit prolonged peristalses that are dependent on GAR-3. Cross talk with GAR-3 contributes to the slow larval growth and extended life span phenotypes observed in eat-2 mutants, indicating that this cross talk has a long-term impact on $C$. elegans feeding.

\section{eat-2 mutants exhibit different effects on pumping and peristalsis}

eat-2 mutants have a reduced pump rate, indicating that EAT-2 plays an excitatory role in pumping (Avery 1993b; Raizen et al. 1995). In comparison, eat-2 mutants exhibit prolonged peristalses, indicating that EAT-2 does not excite isthmus muscle contractions. Rather, it is necessary for rapid relaxation. These peristalsis phenotypes are similar to previous observations demonstrating that eat-2 mutation leads to prolonged depolarization and contraction of the terminal bulb muscles during pumping (Steger and Avery 2004).

The EAT-2 receptor is specifically localized at the sites where MC forms synapses near the junction of the pm4 and pm5 pharyngeal muscles, and it is necessary for MC to directly stimulate rapid pumping (McKay et al. 2004). Because MC does not synapse on either the posterior isthmus or the terminal bulb (Albertson and Thomson 1976; McKay et al. 2004), the prolonged contraction of these muscles is likely an indirect effect of loss of EAT-2-containing receptors.

\section{GAR-3 stimulates prolonged peristalsis in eat-2 mutants}

The prolonged peristalses in eat- 2 mutants depend on the GAR-3 receptor. gar-3 mutation partially suppressed the prolonged peristaltic contraction of eat-2 mutants, as well as the increased and prolonged $\mathrm{Ca}^{2+}$ transients in the isthmus muscles of these animals. This suppression demonstrates that GAR-3 receptor activation contributes to the eat-2 mutant 

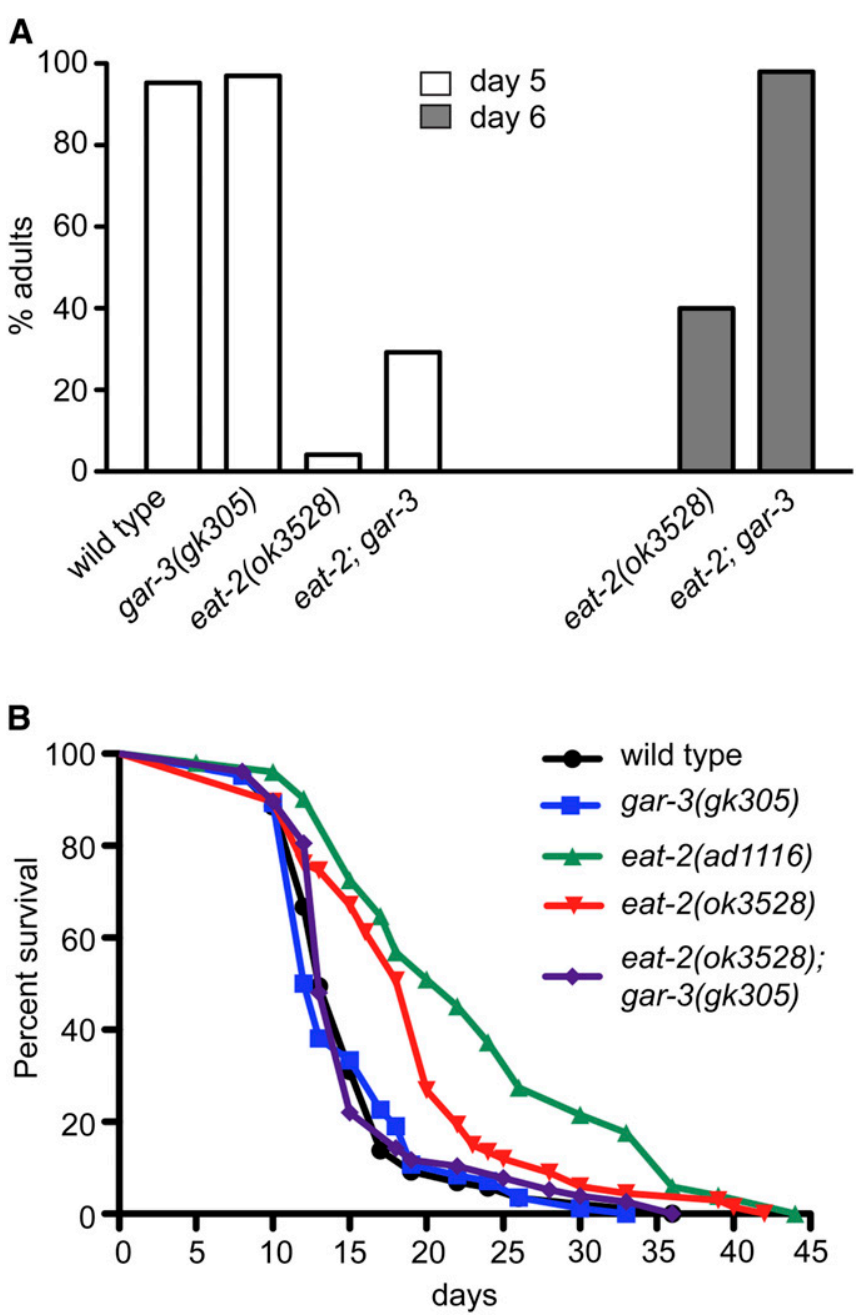

Figure 9 gar-3 mutation suppresses the slow growth and prolonged life span of eat-2 mutants. (A) Bar graphs indicating the percent animals of the indicated genotypes reaching adulthood by day 5 or day 6 at $20^{\circ}(n=$ 100 for each genotype). (B) Adult survival curves for animals of the indicated genotypes. Averages of representative triplicate assays performed on plates containing UV-killed $E$. coli as a food source. Nearly identical results were obtained in assays with living $E$. coli.

peristaltic phenotypes. Previous studies demonstrated that gar-3 mutation similarly suppressed the prolonged contraction of the terminal bulb muscles in eat-2 mutants (Steger and Avery 2004). In comparison, gar-3 single mutants have relatively minor defects in peristalsis. The duration of peristalsis was similar to wild-type animals. However, these mutants exhibited a sharp decrease in the peak delay between $\mathrm{Ca}^{2+}$ transients in the center and posterior isthmus, and a decreased $\Delta \mathrm{F} / \mathrm{F}_{\mathrm{o}}$ in the posterior isthmus. Thus, in wild-type animals, GAR-3 contributes to the spatially and temporally dynamic changes in $\mathrm{Ca}^{2+}$ concentration in the isthmus muscles, but this did not produce recognizable changes in the duration of peristalsis. These observations demonstrate that GCaMP3 may be a more sensitive assay for muscle excitation.

In contrast to its effect on peristalsis, gar-3 mutation did not suppress the reduced pump rate observed in eat- 2 mutants
(Steger and Avery 2004). Thus, the pumping and peristalsis defects in eat-2 mutants arise via different mechanisms. The reduced pump rate results directly from a loss of EAT-2 excitatory activity stimulated by the MC neuron, while the prolonged peristalsis results from excitation that depends on GAR-3.

\section{ACh spillover may produce prolonged peristalses in eat-2 mutants}

Extrasynaptic GAR-3 has previously been shown to respond to humoral ACh (Chan et al. 2013) and we suggest that in eat-2 mutants, ACh spillover from synapses between MC near the junction of the pm4 and pm5 muscles similarly activates GAR-3 receptors in pm5. Activation of slow-acting, metabotropic GAR-3 stimulates increased and prolonged $\mathrm{Ca}^{2+}$ transients in the isthmus, which lead to prolonged peristalsis. GAR-3 is known to regulate $\mathrm{Ca}^{2+}$-dependent processes in the pharyngeal muscles and increased GAR-3 signaling produces prolonged pharyngeal muscle contractions during pumping (Steger and Avery 2004). Because gar-3 mutation only partially suppresses the prolonged peristalsis in eat- 2 mutants, other mechanisms must also contribute to it. For example, voltage-activated $\mathrm{K}^{+}$channels such as EXP-2 might not be efficiently activated in eat-2 mutants for rapid repolarization of the isthmus muscles (Davis et al. 1999; Shtonda and Avery 2005).

At cholinergic synapses, unbound ACh is normally degraded by acetylcholinesterases located at the synapse [reviewed in Rotundo (2003)]. However, ACh released at mammalian NMJs can coactivate mAChRs to stimulate vasodilation in nearby blood vessels (Welsh and Segal 1997), although the physiological significance of this stimulation is uncertain (Hong and Kim 2017). ACh spillover has also been observed when quantal content is increased or when acetylcholinesterases are inhibited (Stanchev and Sargent 2011; Petrov et al. 2014). We have similarly observed that $C$. elegans ace- 3 mutants with reduced acetylcholinesterase activity produce prolonged peristalses that are dependent on GAR-3.

While MC morphology and synapse positioning is normal in eat-2 mutants, we cannot rule out that other defects in synapse formation or remodeling occur in these animals. Interestingly, loss of the $\gamma$-subunit of the nAChR leads to a diffuse distribution of acetylcholinesterase clusters during the development of mouse diaphragm muscles (Liu et al. 2008), and a similar defect in acetylcholinesterase localization could underlie signaling from the MC to the pharyngeal isthmus muscles in eat-2 mutants.

Increased cytoplasmic $\mathrm{Ca}^{2+}$ levels in muscle cells have been observed in a number of human neuromuscular disorders, including myotonic dystrophies, Duchenne muscular dystrophy, as well as in ColQ and slow-channel myasthenic syndromes [reviewed in Vallejo-Illarramendi et al. (2014), Engel et al. (2015)]. In particular, myotonic dystrophies are initially characterized by the delayed relaxation of muscles after contraction, and this resembles the prolonged peristalsis that we have observed in eat- 2 and other mutants. Thus, 
eat-2 mutants may provide a new model to characterize the immediate effects of $\mathrm{Ca}^{2+}$ dysregulation in muscle cells.

\section{Acknowledgments}

The authors are indebted to Paul Huber, Angenee Milton, Zhihua Li, Janet Richmond, Sreekanth Chalasani, Sebnem Ece Eksi, Ian Hope, and many Molecular, Cell, and Developmental Biology colleagues for reagents, strains, and advice. Some strains were provided by the Caenorhabditis Genetics Center, which is funded by the National Institutes of Health (NIH) Office of Research Infrastructure Programs (P40 OD-010440). This project was supported by the NIH/National Institute of General Medical Sciences (R01 GM-82865), a University of Illinois at Chicago (UIC) Campus Research Board Pilot grant, and a UIC Liberal Arts and Sciences Award for Faculty in the Natural Sciences.

\section{Literature Cited}

Albertson, D. G., and J. N. Thomson, 1976 The pharynx of Caenorhabditis elegans. Philos. Trans. R. Soc. Lond. B Biol. Sci. 275: 299-325. https://doi.org/10.1098/rstb.1976.0085

Albuquerque, E. X., E. F. Pereira, M. Alkondon, and S. W. Rogers, 2009 Mammalian nicotinic acetylcholine receptors: from structure to function. Physiol. Rev. 89: 73-120. https://doi.org/ 10.1152/physrev.00015.2008

Ausubel, F. M., 1990 Current Protocols in Molecular Biology. WileyInterscience, New York.

Avery, L., 1993a The genetics of feeding in Caenorhabditis elegans. Genetics 133: 897-917.

Avery, L., 1993b Motor neuron M3 controls pharyngeal muscle relaxation timing in Caenorhabditis elegans. J. Exp. Biol. 175: 283-297.

Avery, L., and H. R. Horvitz, 1987 A cell that dies during wild-type C. elegans development can function as a neuron in a ced-3 mutant. Cell 51: 1071-1078. https://doi.org/10.1016/00928674(87)90593-9

Avery, L., and H. R. Horvitz, 1989 Pharyngeal pumping continues after laser killing of the pharyngeal nervous system of C. elegans. Neuron 3: 473-485. https://doi.org/10.1016/08966273(89)90206-7

Avery, L., and H. R. Horvitz, 1990 Effects of starvation and neuroactive drugs on feeding in Caenorhabditis elegans. J. Exp. Zool. 253: 263-270. https://doi.org/10.1002/jez.1402530305

Avery, L., and Y. J. You, 2012 C. elegans feeding (May 21, 2012), WormBook, ed. The C. elegans Research Community, WormBook, doi/10.1895/wormbook.1.150.1, http://www.wormbook.org.

Beaster-Jones, L., and P. G. Okkema, 2004 DNA binding and in vivo function of $\mathrm{C}$. elegans PEB-1 require a conserved FLYWCH motif. J. Mol. Biol. 339: 695-706. https://doi.org/ 10.1016/j.jmb.2004.04.030

Bers, D. M., 2002 Cardiac excitation-contraction coupling. Nature 415: 198-205. https://doi.org/10.1038/415198a

Brenner, S., 1974 The genetics of Caenorhabditis elegans. Genetics 77: 71-94.

C. elegans Deletion Mutant Consortium, 2012 Large-scale screening for targeted knockouts in the Caenorhabditis elegans genome. G3 (Bethesda) 2: 1415-1425. https://doi.org/10.1534/ g3.112.003830

Chalasani, S. H., N. Chronis, M. Tsunozaki, J. M. Gray, D. Ramot et al., 2007 Dissecting a circuit for olfactory behaviour in
Caenorhabditis elegans. Nature 450: 63-70 [corrigenda: Nature 451: 102 (2008)]; [corrigenda: Nature 533: 130 (2016)]. https://doi.org/10.1038/nature06292

Chan, J. P., T. A. Staab, H. Wang, C. Mazzasette, Z. Butte et al., 2013 Extrasynaptic muscarinic acetylcholine receptors on neuronal cell bodies regulate presynaptic function in Caenorhabditis elegans. J. Neurosci. 33: 14146-14159. https://doi.org/ 10.1523/JNEUROSCI.1359-13.2013

Combes, D., Y. Fedon, M. Grauso, J. P. Toutant, and M. Arpagaus, 2000 Four genes encode acetylcholinesterases in the nematodes Caenorhabditis elegans and Caenorhabditis briggsae. cDNA sequences, genomic structures, mutations and in vivo expression. J. Mol. Biol. 300: 727-742. https://doi.org/10.1006/ jmbi.2000.3917

Culetto, E., D. Combes, Y. Fedon, A. Roig, J. P. Toutant et al., 1999 Structure and promoter activity of the 5 ' flanking region of ace-1, the gene encoding acetylcholinesterase of class A in Caenorhabditis elegans. J. Mol. Biol. 290: 951-966. https://doi. org/10.1006/jmbi.1999.2937

Davis, M. W., R. Fleischhauer, J. A. Dent, R. H. Joho, and L. Avery, 1999 A mutation in the C. elegans EXP-2 potassium channel that alters feeding behavior. Science 286: 2501-2504. https:// doi.org/10.1126/science.286.5449.2501

Dittman, J. S., and J. M. Kaplan, 2008 Behavioral impact of neurotransmitter-activated G-protein-coupled receptors: muscarinic and GABAB receptors regulate Caenorhabditis elegans locomotion. J. Neurosci. 28: 7104-7112. https://doi.org/10.1523/ JNEUROSCI.0378-08.2008

Engel, A. G., X. M. Shen, D. Selcen, and S. M. Sine, 2015 Congenital myasthenic syndromes: pathogenesis, diagnosis, and treatment. Lancet Neurol. 14: 420-434. https://doi.org/10.1016/S14744422(14)70201-7

Feng, H., and I. A. Hope, 2013 The Caenorhabditis elegans homeobox gene ceh-19 is required for MC motorneuron function. Genesis 51: 163-178. https://doi.org/10.1002/dvg.22365

Gilhus, N. E., 2016 Myasthenia gravis. N. Engl. J. Med. 375: 2570-2581. https://doi.org/10.1056/NEJMra1602678

Hong, K. S., and K. Kim, 2017 Skeletal muscle contraction-induced vasodilation in the microcirculation. J. Exerc. Rehabil. 13: 502507. https://doi.org/10.12965/jer.1735114.557

Johnson, C. D., J. B. Rand, R. K. Herman, B. D. Stern, and R. L. Russell, 1988 The acetylcholinesterase genes of C. elegans: identification of a third gene (ace-3) and mosaic mapping of a synthetic lethal phenotype. Neuron 1: 165-173. https://doi. org/10.1016/0896-6273(88)90201-2

Kamentsky, L., T. R. Jones, A. Fraser, M. A. Bray, D. J. Logan et al., 2011 Improved structure, function and compatibility for CellProfiler: modular high-throughput image analysis software. Bioinformatics 27: 1179-1180. https://doi.org/10.1093/ bioinformatics/btr095

Kerr, R. A., 2006 Imaging the activity of neurons and muscles (June 2, 2006), WormBook, ed. The C. elegans Research Community, WormBook, doi/10.1895/wormbook.1.113.1, http:// www.wormbook.org.

Lakowski, B., and S. Hekimi, 1998 The genetics of caloric restriction in Caenorhabditis elegans. Proc. Natl. Acad. Sci. USA 95: 13091-13096. https://doi.org/10.1073/pnas.95.22.13091

Lee, Y. S., Y. S. Park, S. Nam, S. J. Suh, J. Lee et al., 2000 Characterization of GAR-2, a novel G protein-linked acetylcholine receptor from Caenorhabditis elegans. J. Neurochem. 75: 1800-1809. https://doi.org/10.1046/j.1471-4159.2000. 0751800.x

Lewis, J. A., and J. T. Fleming, 1995 Basic culture methods, pp. 4-30 in Methods in Cell Biology-Caenorhabditis elegans: Modern Biological Analysis of an Organism. Academic Press, San Diego.

Liu, Y., B. LeBoeuf, and L. R. Garcia, 2007 G alpha(q)-coupled muscarinic acetylcholine receptors enhance nicotinic acetylcholine 
receptor signaling in Caenorhabditis elegans mating behavior. $\mathrm{J}$. Neurosci. 27: 1411-1421. https://doi.org/10.1523/JNEUROSCI. 4320-06.2007

Liu, Y., D. Padgett, M. Takahashi, H. Li, A. Sayeed et al., 2008 Essential roles of the acetylcholine receptor gammasubunit in neuromuscular synaptic patterning. Development 135: 1957-1967. https://doi.org/10.1242/dev.018119

McKay, J. P., D. M. Raizen, A. Gottschalk, W. R. Schafer, and L. Avery, 2004 eat- 2 and eat-18 are required for nicotinic neurotransmission in the Caenorhabditis elegans pharynx. Genetics 166: 161-169. https://doi.org/10.1534/genetics.166.1.161

Mello, C., and A. Fire, 1995 DNA transformation, pp. 451-482 in Caenorhabditis elegans: Modern Biological Analysis of an Organism, edited by H. F. Epstein, and D. C. Shakes. Academic Press, San Diego. https://doi.org/10.1016/S0091-679X(08)61399-0

Nonet, M. L., 1999 Visualization of synaptic specializations in live C. elegans with synaptic vesicle protein-GFP fusions. J. Neurosci. Methods 89: 33-40. https://doi.org/10.1016/S0165-0270 (99)00031-X

Okkema, P. G., S. W. Harrison, V. Plunger, A. Aryana, and A. Fire, 1993 Sequence requirements for myosin gene expression and regulation in Caenorhabditis elegans. Genetics 135: 385-404.

Pereira, L., P. Kratsios, E. Serrano-Saiz, H. Sheftel, A. E. Mayo et al., 2015 A cellular and regulatory map of the cholinergic nervous system of C. elegans. Elife 4: e12432. https://doi.org/10.7554/ eLife.12432

Petrov, K. A., E. Girard, A. D. Nikitashina, C. Colasante, V. Bernard et al., 2014 Schwann cells sense and control acetylcholine spillover at the neuromuscular junction by alpha7 nicotinic receptors and butyrylcholinesterase. J. Neurosci. 34: 1187011883. https://doi.org/10.1523/JNEUROSCI.0329-14.2014

Raizen, D. M., and L. Avery, 1994 Electrical activity and behavior in the pharynx of Caenorhabditis elegans. Neuron 12: 483-495. https://doi.org/10.1016/0896-6273(94)90207-0

Raizen, D. M., R. Y. Lee, and L. Avery, 1995 Interacting genes required for pharyngeal excitation by motor neuron MC in Caenorhabditis elegans. Genetics 141: 1365-1382.

Ramakrishnan, K., and P. G. Okkema, 2014 Regulation of C. elegans neuronal differentiation by the ZEB-family factor ZAG-1 and the NK-2 homeodomain factor CEH-28. PLoS One 9: e113893. https://doi.org/10.1371/journal.pone.0113893

Rand, J. B., 1989 Genetic analysis of the cha-1-unc-17 gene complex in Caenorhabditis. Genetics 122: 73-80.

Ray, P., R. Schnabel, and P. G. Okkema, 2008 Behavioral and synaptic defects in C. elegans lacking the NK-2 homeobox gene ceh-28. Dev. Neurobiol. 68: 421-433. https://doi.org/10.1002/ dneu.20599

Rotundo, R. L., 2003 Expression and localization of acetylcholinesterase at the neuromuscular junction. J. Neurocytol. 32: 743766. https://doi.org/10.1023/B:NEUR.0000020621.58197.d4

Ruaud, A. F., and J. L. Bessereau, 2006 Activation of nicotinic receptors uncouples a developmental timer from the molting timer in C. elegans. Development 133: 2211-2222. https:// doi.org/10.1242/dev.02392

Saur, T., S. E. DeMarco, A. Ortiz, G. R. Sliwoski, L. Hao et al., 2013 A genome-wide RNAi screen in Caenorhabditis elegans identifies the nicotinic acetylcholine receptor subunit ACR-7 as an antipsychotic drug target. PLoS Genet. 9: e1003313. https:// doi.org/10.1371/journal.pgen.1003313

Schindelin, J., I. Arganda-Carreras, E. Frise, V. Kaynig, M. Longair et al., 2012 Fiji: an open-source platform for biological-image analysis. Nat. Methods 9: 676-682. https://doi.org/10.1038/ nmeth.2019

Shimozono, S., T. Fukano, K. D. Kimura, I. Mori, Y. Kirino et al., 2004 Slow Ca2+ dynamics in pharyngeal muscles in Caenorhabditis elegans during fast pumping. EMBO Rep. 5: 521-526. https://doi.org/10.1038/sj.embor.7400142

Shtonda, B., and L. Avery, 2005 CCA-1, EGL-19 and EXP-2 currents shape action potentials in the Caenorhabditis elegans pharynx. J. Exp. Biol. 208: 2177-2190. https://doi.org/10.1242/ jeb.01615

Song, B. M., and L. Avery, 2012 Serotonin activates overall feeding by activating two separate neural pathways in Caenorhabditis elegans. J. Neurosci. 32: 1920-1931. https://doi.org/ 10.1523/JNEUROSCI.2064-11.2012

Stanchev, D., and P. B. Sargent, 2011 alpha7-Containing and nonalpha7-containing nicotinic receptors respond differently to spillover of acetylcholine. J. Neurosci. 31: 14920-14930. https:// doi.org/10.1523/JNEUROSCI.3400-11.2011

Starich, T. A., R. Y. Lee, C. Panzarella, L. Avery, and J. E. Shaw, 1996 eat-5 and unc-7 represent a multigene family in Caenorhabditis elegans involved in cell-cell coupling. J. Cell Biol. 134: 537-548. https://doi.org/10.1083/jcb.134.2.537

Steger, K. A., and L. Avery, 2004 The GAR-3 muscarinic receptor cooperates with calcium signals to regulate muscle contraction in the Caenorhabditis elegans pharynx. Genetics 167: 633-643. https://doi.org/10.1534/genetics.103.020230

Steger, K. A., B. B. Shtonda, C. Thacker, T. P. Snutch, and L. Avery, 2005 The C. elegans T-type calcium channel CCA-1 boosts neuromuscular transmission. J. Exp. Biol. 208: 2191-2203. https://doi.org/10.1242/jeb.01616

Stuyvers, B. D., P. A. Boyden, and H. E. ter Keurs, 2000 Calcium waves: physiological relevance in cardiac function. Circ. Res. 86: 1016-1018. https://doi.org/10.1161/01.RES.86.10.1016

Sutphin, G. L., and M. Kaeberlein, 2009 Measuring Caenorhabditis elegans life span on solid media. J. Vis. Exp. 27: 1152. https://doi.org/10.3791/1152

Talesa, V., E. Culetto, N. Schirru, H. Bernardi, Y. Fedon et al., 1995 Characterization of a null mutation in ace-1, the gene encoding class A acetylcholinesterase in the nematode Caenorhabditis elegans. FEBS Lett. 357: 265-268. https://doi.org/ 10.1016/0014-5793(94)01343-Y

Thevenaz, P., U. E. Ruttimann, and M. Unser, 1998 A pyramid approach to subpixel registration based on intensity. IEEE Trans. Image Process. 7: 27-41. https://doi.org/10.1109/83.650848

Tian, L., S. A. Hires, T. Mao, D. Huber, M. E. Chiappe et al., 2009 Imaging neural activity in worms, flies and mice with improved GCaMP calcium indicators. Nat. Methods 6: 875881. https://doi.org/10.1038/nmeth.1398

Vallejo-Illarramendi, A., I. Toral-Ojeda, G. Aldanondo, and A. Lopez de Munain, 2014 Dysregulation of calcium homeostasis in muscular dystrophies. Expert Rev. Mol. Med. 16: e16. https:// doi.org/10.1017/erm.2014.17

Welsh, D. G., and S. S. Segal, 1997 Coactivation of resistance vessels and muscle fibers with acetylcholine release from motor nerves. Am. J. Physiol. 273: H156-H163.

Wess, J., 2004 Muscarinic acetylcholine receptor knockout mice: novel phenotypes and clinical implications. Annu. Rev. Pharmacol. Toxicol. 44: 423-450. https://doi.org/10.1146/annurev. pharmtox.44.101802.121622

Communicating editor: B. Grant 